\title{
Questions politiques et développement durable au centre des problèmes de cohérence
}

Rapport de synthèse

\section{Monika Egger et Jacques Forster}

\section{(QpenEdition}

\section{Journals}

Édition électronique

URL : http://journals.openedition.org/aspd/1484

DOI : $10.4000 /$ aspd. 1484

ISSN : 1663-9669

\section{Éditeur}

Institut de hautes études internationales et du développement

Édition imprimée

Date de publication : 1 février 1995

Pagination : 203-217

ISSN : 1660-5934

\section{Référence électronique}

Monika Egger et Jacques Forster, «Questions politiques et développement durable au centre des problèmes de cohérence », Annuaire suisse de politique de développement [En ligne], 14 | 1995, mis en ligne le 04 mai 2013, consulté le 08 septembre 2020. URL : http://journals.openedition.org/aspd/1484 ; DOI : https://doi.org/10.4000/aspd.1484 


\title{
Questions politiques et développement durable au centre des problèmes de cohérence
}

\section{Rapport de synthèse}

\author{
Monika Egger, Jacques Forster \\ Cohérence: Liaison, rapport étroit d'idées qui s'accordent entre elles. \\ (Le Grand Robert) \\ "Absence de contradiction et de disparate entre les parties \\ d'un argument, d'une doctrine, d'un ouvrage.»
}

(Lalande)

Lorsque l'on s'interroge sur la cohérence ou l'incohérence des relations bilatérales de la Suisse avec l'Indonésie, on remarque d'emblée à quel point cette problématique est complexe et les niveaux d'action et d'intervention nombreux. La présentation schématique (voir tableau page suivante) des problèmes, tels qu'ils ont été abordés par les divers participants au colloque, illustre cette réalité. Les problèmes de cohérence identifiés en Suisse et en Indonésie y sont classés selon les objectifs de la politique étrangère.

Comme toujours lorsqu'un problème se révèle complexe, la tâche la plus ardue dans l'examen de la cohérence a consisté à fixer des priorités. C'est en effet le choix du principal problème de cohérence qui est à l'origine du colloque: Quel est, à mon avis, le principal problème de cohérence dans les relations entre la Suisse et l'Indonésie? Quels sont, selon moi, les interlocuteurs et les acteurs à même de résoudre le principal problème de cohérence?" De plus, il ne faut pas perdre de vue, qu'en corollaire à ces questions, on se demande aussi quels espoirs on place dans une cohérence accrue dans un secteur-clé et quelle contribution concrète l'on peut fournir pour optimiser les actions.

La majorité des participants au séminaire ont cité deux grands thèmes: un de nature politique et un de nature économique:

1. Démocratie, processus de démocratisation, bonne gestion gouvernementale (Good Governance), situation des droits de l'homme en Indonésie;

2. Instauration d'une croissance économique durable dans le pays. 
Les niveaux d'action étant interdépendants, une amélioration de la cohérence exige l'intervention de tous les acteurs mentionnés dans le tableau. II convient par ailleurs de rechercher, davantage que par le passé, le concours d'autres partenaires que celui du gouvernement central.

\section{Les problèmes de cohérence dans les objectifs de la politique extérieure de la Suisse et les acteurs concernés}

\begin{tabular}{|c|c|c|}
\hline & INDONESIE & SUISSE \\
\hline $\begin{array}{l}\text { Sécurité et paix, } \\
\text { Etat de droit/ démocratie } \\
\text { Droits de l'homme }\end{array}$ & $\begin{array}{l}\text { - Corruption } \\
\text { - Etat autoritaire } \\
\text { - Droits de l'homme } \\
\text { - Conflits ethniques et } \\
\text { religieux } \\
\text { - "Good governance" } \\
\text { - Contrôle des armements }\end{array}$ & $\begin{array}{l}\text { - Exportations d'armes } \\
\text { - Aide judiciaire } \\
\text { - «Good governance» } \\
\text { - Contrôle des armement }\end{array}$ \\
\hline Economie et travail & $\begin{array}{l}\text { Economie duale } \\
\text { Marché du travail } \\
\text { Droits syndicaux }\end{array}$ & $\begin{array}{l}\text { - Commerce et investisse- } \\
\text { ments directs } \\
\text { - Clauses sociales } \\
\text { - Coopération au } \\
\text { développement } \\
\text { - Garantie contre les } \\
\text { risques à l'exportation } \\
\text { - Fuite de capitaux } \\
\text { - Droit des brevets } \\
\text { - Conventions de double } \\
\text { imposition }\end{array}$ \\
\hline $\begin{array}{l}\text { Lutte contre les inégalités } \\
\text { sociale }\end{array}$ & $\begin{array}{l}\text { - Promotion équilibrée } \\
\text { hommes-femmes } \\
\text { - Stratégie de lutte } \\
\text { contre la pauvreté }\end{array}$ & $\begin{array}{l}\text { - Coopération au } \\
\text { développement } \\
\text { - Stratégie de lutte } \\
\text { contre la pauvreté }\end{array}$ \\
\hline Environnement & $\begin{array}{l}\text { - Surexploitation } \\
\text { des forêts tropicales } \\
\text { - Politique énergétique } \\
\text { - Destruction de } \\
\text { l'environnement }\end{array}$ & $\begin{array}{l}\text { - Politique énergétique } \\
\text { - Boycott des bois tropicaux } \\
\text { - Destructions de } \\
\text { l'environnement }\end{array}$ \\
\hline
\end{tabular}

Gouvernement/administration/armée

Economie privée/syndicats

Oeuvres d'entraide/ONG/Eglises

Scientifiques

Partenaires multilateraux (ONU, Union de l'Europe, FMI/Banque mondiale, ASEAN)

Autres partenaires bilatéraux 
"Jusqu'à présent, nous avons surtout collaboré avec le gouvernement central. II me paraît important que nous recherchions dorénavant d'autres partenaires, que l'économie privée intervienne davantage en tant que partenaire - non seulement dans le secteur des exportations en provenance de Suisse, mais aussi dans le domaine des investissements privés - que l'économie suisse s'intéresse plus à créer des places de travail aussi en Indonésie" (Robert Jenny). "La situation des femmes mérite d'être renforcée. J'attends beaucoup de la "promotion équilibrée hommes-femmes menée par les ONG. Je pense que les organes étatiques sont encore très, très loin de la réalité" (Margrit Meier).

Le poids accordé aux problèmes et les éventuelles amorces de solution varient selon les points de vue: les participants ont exprimé une opinion unanime - pour ne pas dire harmonieuse - sur les questions politiques (situation des droits de l'homme) en Indonésie, ainsi que sur la nécessité de promouvoir la démocratie et d'améliorer la gestion gouvernementale. Les interventions concernant la cohérence dans les relations économiques de la Suisse avec l'Indonésie, dans la perspective du développement durable, ont été plus contrastées et passionnées. Pour ce qui est du développement durable, l'accent a été mis sur une croissance économique socialement mieux équilibrée, tandis que les problèmes liés à l'environnement n'ont guère été approfondis.

\section{Améliorer la politique pour accroître la cohérence - démocratisation, bonne gestion gouvernementale, droits de l'homme}

\section{Relations bilatérales}

Le débat sur la dimension politique des problèmes de cohérence a été marqué par la décision prise quelques jours auparavant par les Etats-Unis de dissocier totalement, dans leurs relations avec la Chine, la question des droits de l'homme et la politique commerciale. Les participants au colloque se trouvaient donc d'entrée de jeu face à la question de savoir si la Suisse devait rompre ses relations économiques avec un pays lorsque celui-ci ne respecte pas les droits de l'homme. Et cette autre question: I'Indonésie fait-elle partie de ce type de pays?

“Nous ne disposons d'aucun mécanisme très subtil pour apaiser les tensions pouvant surgir entre le secteur des droits de l'homme et la politique économique extérieure. Dans la pratique, on passe longtemps les éventuelles difficultés sous silence mais, face à un problème d'incohérence, on ne connaît guère que deux types de réactions: les uns exigent la rupture immédiate des relations économiques, par exemple suite à une violation grave des droits de l'homme, tandis que les représentants de l'économie expliquent, pour leur part, pourquoi il ne faut pas le faire" (Peter Maurer). 
Pour éviter que l'opinion publique soit partagée en deux camps opposés pour ce qui est des relations avec des gouvernements politiquement suspects, il faut instaurer un dialogue plus large. Ce dialogue devrait intervenir régulièrement dans le cadre d'échanges plus fréquents entre les ONG, l'industrie et l'administration, et avoir pour but de doter la Suisse d'une politique étrangère et d'une politique de développement cohérentes face à des violations des droits de l'homme. On pourrait ainsi définir une position de principe qui pourrait être adaptée à la situation spécifique d'un pays. Le cas échéant, on pourrait ensuite faire des distinctions selon la situation et selon le pays concerné, en veillant toutefois à ne pas appliquer des normes différentes aux divers pays en fonction des seuls intérêts de la Suisse, de l'importance de ses relations économiques et de l'aide au développement accordée. "Je pense qu'il faut néanmoins prendre garde à ne pas appliquer des critères différents uniquement parce que l'on a plus d'influence dans certains pays" (Hans- Peter Maag).

La majorité des participants au colloque pensent qu'un petit pays comme la Suisse ne peut guère exercer d'influence politique sur un pays comme I'Indonésie. "L'Indonésie compte certes parmi les pays de concentration de la coopération au développement, mais les Indonésiens ne considèrent de loin pas la Suisse comme un partenaire important, ni sur le plan de la coopération au développement ni sur le plan politique. De plus, I'Indonésie fait partie des pays qui peuvent se permettre d'ignorer les conditions qu'on voudrait leur imposer ou toute pression internationale en matière de droits de l'homme, de bonne gestion gouvernementale ou de démocratie." (Konrad Specker)

Bien que les participants relativisent les résultats que la Suisse pourrait obtenir, ils estiment tous qu'elle devrait s'efforcer de mener une politique économique extérieure qui ne fasse pas abstraction des atteintes aux droits de l'homme. Même si nos interventions ne peuvent avoir que des effets limités, nous ne devons pas nous laisser aller à la résignation et à l'inaction. II faut empêcher les exportations suisses lorsqu'elles favorisent les violations des droits de l'homme (exportations d'armes). En général, il faut veiller plus à ce que les relations économiques de la Suisse ne profitent pas à des pouvoirs corrompus et n'entravent ainsi l'essor de la démocratie. Pour cerner les besoins d'un pays, il suffit de demander aux personnes concernées par nos relations économiques et par l'aide au développement, comment elles interprètent notre propre notion des relations économiques, de la bonne gestion gouvernementale et des droits de l'homme: "Qu'attendez-vous de la Suisse? Des organisations d'entraide suisses? De l'Etat? De l'économie privée? Nous ne devrions pas craindre de poser ces questions. L'expérience m'a en effet montré que - les sensibilités étant différentes - les réponses sont en général plus nuancées qu'un simple appel au boycott, par exemple" (Richard Gerster).

En améliorant le dialogue avec nos partenaires privés et publics en Indonésie, à tous les échelons - gouvernement à la capitale, mais aussi dans les régions et districts périphériques - nous faisons connaître notre notion de la démocratie et notre culture dans d'autres Etats. En obtenant de meilleures 
informations en provenance directe des diverses couches de la population, nous pourrons nous faire une meilleure idée de la situation politique et mettre en oeuvre nos moyens de l'améliorer. Nous devrons aussi adapter à la situation de chaque pays notre conception de la croissance économique idéale. La théorie présentée par Jean-Luc Maurer, selon laquelle il faut tout d'abord un Etat autoritaire pour parvenir à un certain niveau de développement, niveau à partir duquel des forces démocratiques naissent d'elles-mêmes, a soulevé des controverses. Cette théorie est contestée. D'aucuns ont en effet rappelé le cas de l'Europe où les sociétés ont suivi parallèlement un développement démocratique et économique. "Le développement économique, l'élévation du niveau de vie et la démocratie sont des éléments interdépendants. II existe des liens complexes entre démocratie et prospérité, mais elles se stimulent l'une l'autre"' (Günther Bächler).

Les violations des droits de l'homme ne doivent pas être passées sous silence; elles doivent être abordées au niveau diplomatique. De son côté, l'économie privée devrait également se doter de moyens pour exprimer - non pas au niveau d'entreprises individuelles, mais dans le cadre d'une association professionnelle - sa désapprobation face à des cas concrets d'atteintes aux droits de l'homme. Elle devrait expliquer à ses partenaires qu'à long terme ces atteintes vont à l'encontre des principes qui prévalent normalement dans les relations économiques. Si l'Indonésie souhaite attirer un plus grand volume d'investissements étrangers, elle a en fin de compte tout intérêt à donner l'image d'un pays politiquement stable. Or le respect des droits de l'homme est une condition fondamentale de stabilité politique à long terme. Ce point de vue contredit l'opinion largement répandue dans les milieux économiques, qui veut qu'un système répressif n'engendre pas automatiquement un mauvais climat pour les investissements privés, du moins à court terme. Face au choix, les investisseurs préféreront probablement des systèmes répressifs à des systèmes démocratiques peu sûrs et trop bureaucratiques. C'est un élément à prendre en considération lorsque l'on aborde le rôle de l'économie privée dans l'accroissement de la cohérence. Pour avancer sur cette voie, il faudra cependant fournir encore beaucoup d'efforts de persuasion. En effet, pour l'économie privée, une augmentation des dépenses militaires peut être synonyme du maintien - par la répression - de l'ordre et du calme, donc de la sécurité des investissements.

La politique des droits de l'homme ne peut se cantonner dans le rôle de bonne conscience de la coopération économique. Elle doit aussi se concrétiser dans les faits. Les mesures positives ne doivent pas se limiter aux effets indirects de la croissance économique. Une mesure positive concrète consisterait par exemple à élaborer et à appliquer un code de conduite de l'industrie suisse dans la démocratisation d'un pays. Celui-ci fixerait la contribution de l'économie privée à la coopération au développement, une orientation identique pour les relations bilatérales privées et publiques, etc. Dans l'ensemble, on souhaite que la Suisse se montre un peu moins "hypocrite" et amorce un "tournant fondamental» dans sa pensée politique (Robert Jenny). 
Au demeurant, nous ne devons pas faire abstraction de l'histoire de notre propre développement et des injustices qui le jalonnent. Les excès de langage sont aussi à éviter. "Le "régime autoritaire" en Indonésie est-il comparable à celui du Chili de Pinochet ou à celui de l'Espagne de Franco? Certes, la corruption existe, mais il ne faut pas oublier que la corruption sévit aussi en Suisse et ailleurs" (Philippe Régnier).

La crédibilité des pays du Nord dans le cadre de leur dialogue politique et économique avec les pays du Sud joue un rôle central dans le débat sur la cohérence. Sommes-nous crédibles - nous qui consommons de l'énergie sans compter - lorsque nous leur expliquons qu'ils ne parviendront pas à instaurer un développement durable sans juguler leur consommation énergétique? La Suisse est-elle crédible lorsqu'elle adhère aux sanctions économiques dans un cas (Haïti) et pas dans un autre (Afrique du Sud)? Notre crédibilité nationale est également remise en question en Suisse: "Nos efforts de cohérence sont-ils vraiment crédibles lorsque le peuple rejette en votation toutes les propositions du gouvernement en matière de politique étrangère?" (Robert Jenny). Comment gérons-nous les grandes divergences qui opposent le gouvernement, le parlement et le peuple sur les questions de politique étrangère?

\section{La cohérence dans le contexte international}

Au-delà des relations bilatérales, se posent les questions suivantes: C omment se présente une coopération suisse cohérente avec l'Indonésie dans le contexte international? Que doit faire la Suisse pour adopter une attitude cohérente face à des règles de conduite internationales précises, telles que les sanctions de l'ONU à l'encontre d'un pays (voir les exemples déjà cités de l'Afrique du Sud et de Haiti)?

Des actions coordonnées ou parallèles menées au niveau mondial réussissent parfois à infléchir la politique d'un gouvernement. Une campagne internationale, orchestrée il y a quelques années par des ONG locales en Malaisie a ainsi eu une influence positive sur le processus législatif: le gouvernement a dû abandonner un projet de loi visant à restreindre les droits des organisations privées.

II existe des institutions, telles que le Comité d'aide au développement de I'OCDE ou ie Groupe consultatif de la Banque mondiale, permettant de coordonner, au niveau international, les politiques nationales à l'égard de l'Indonésie. Lorsque les objectifs et les stratégies sont formulées d'un commun accord, on peut en effet escompter une meilleure cohérence.

A long terme, l'interdépendance économique qui prévaut aujourd'hui déjà - et qui ne peut aller que croissant avec le processus de libéralisation n'autorise pas les différents pays à appliquer leurs propres critères dans le domaine des droits de l'homme. "L'interdépendance économique réduira toujours plus la marge de manoeuvre dont dispose l'Etat pour appliquer les droits de l'homme à sa manière. Un pays qui entretient des échanges commerciaux 
intensifs avec des pays qui ne respectent pas les droits syndicaux, ne parviendra guère à les sauvegarder sur son propre territoire. En caricaturant un peu, on peut affirmer ceci: même si le respect des droits des travailleurs en Indonésie nous importe peu du point de vue culturel, nous ne pouvons nous en désintéresser du point de vue économique. Deux système complètement différents ne peuvent pas coexister à long terme" (Margrit Meier).

\section{Une croissance économique durable améliore la cohérence}

En quoi la coopération au développement et la promotion des exportations contribuent-elles à la croissance économique durable en Indonésie? La coopération au développement et la promotion des exportations s'opposent souvent, mais pas toujours. Ce "conflit traditionnel" qui perdure dans la coopération au développement devrait à l'avenir laisser la place à une combinaison d'instruments politiques (policy mix). L'Indonésie constitue un bon exemple de partenaire de la coopération suisse au développement - c'est un pays de concentration de l'aide - où les programmes d'aide technique sont remplacés par des mesures de politique économique. Elle a un besoin urgent de moderniser ses technologies et d'attirer plus d'investissements privés. Si elle souhaite que sa croissance économique se poursuive, l'Indonésie est condamnée à développer ses exportations.

"La promotion des investissements au profit de l'Indonésie - à un moment où le pays se trouve dans une phase de transition entre une économie basée sur la division du travail et une société industrielle en pleine évolution - est un sujet d'importance. Elle offre une occasion rêvée de surmonter l'antagonisme traditionnel entre la coopération au développement et la promotion des exportations, dans la mesure, justement, où il est possible de dynamiser les investissement grâce à des moyens publics. Un tel processus présente des avantages tant pour les entreprises suisses qu'indonésiennes et apporte une contribution économique globale" (Matthias Meyer). Le but de cette démarche est de promouvoir un transfert de technologies efficace, qui puisse contribuer à une croissance économique durable.

La combinaison des instruments politiques implique l'engagement simultané et interactif de divers instruments de coopération et de relations économiques. A ce propos, la majorité des participants au colloque ont exprimé leur désir de voir se poursuivre les programmes de coopération technique de la DDA, car on ne peut concevoir une promotion des technologies sans former les gens. La majorité des participants ne comprennent pas la décision de la DDA de se retirer de l'Indonésie. Au vu des priorités suisses en matière de coopération au développement (lutte contre la pauvreté et soutien des couches de population les plus défavorisées), il importe "d'offrir plus particulièrement quelque chose aux victimes de l'industrialisation et non pas de soutenir la promotion des investissements en général, car cela est à la porté du gouvernement lui-même" (Richard Gerster). 


\section{Plus de cohérence en Indonésie}

Autre point important en matière de cohérence: il convient de demander des explications au Gouvernement indonésien sur ses dépenses militaires (surtout les achats d'avions et autres armes) et de lui proposer des solutions de rechange visant à utiliser ses ressources de manière plus sensée. II faudrait aussi attirer l'attention du Gouvernement indonésien sur sa politique de subventionnement, puisque l'on sait que des moyens financiers considérables sont accordés à des entreprises à bout de souffle appartenant à des protégés du régime. D'énormes déficits sont ainsi couverts par des subsides de l'Etat. Pour l'économie privée suisse, une meilleure cohérence impliquerait par exemple de réduire le volume des affaires conclues avec l'Etat pour accroître ses investissements dans les entreprises privées, de coopérer avec celles-ci et de promouvoir le transfert de technologies adaptées dans le secteur moyen plutôt que dans celui des technologies de pointe. La combinaison des instruments politiques prévoit en effet de soutenir les petites et moyennes entreprises en Indonésie. Elle a également pour objectif d'encourager la collaboration entre entreprises indonésiennes et entreprises suisses travaillant dans le même secteur. Pour commencer, il faudrait toutefois débarrasser les acteurs concernés des inhibitions qu'ils éprouvent face à leurs partenaires potentiels. L'idée de départ repose sur un constat général: jusqu'ici, on a sous-estimé le rôle que peut jouer le secteur privé dans la coopération traditionnelle au développement. Forte de ce constat, l'OFAEE accorde désormais la priorité à la promotion des techniques de l'environnement et d'une formation prise en charge par le secteur privé. A l'avenir, les crédits mixtes perdront sans doute de leur importance dans la coopération au développement avec l'Indonésie.

\section{Qu'est-ce que la cohérence?}

La discussion portant sur les questions politiques et sociales, d'une part, et sur le développement économique durable, d'autre part, revenait toujours au problème de la cohérence, et il se peut que les participants ne donnaient pas tous le même sens à ce terme. C'est pourquoi la troisième partie des débats a été axée sur les questions suivantes: "Qu'entendez-vous par cohérence?" et "Qu'attendez-vous de ce processus de dialogue commun - amorcé par la publication des Lignes directrices Nord-Sud - qui a pour but d'accroître la cohérence?»

Des réponses aussi courtes et percutantes que possible, devaient éclairer le sens donné à ce colloque-test, c'est-à-dire évaluer l'utilité d'un dialogue sur des perspectives communes entre des acteurs ayant des intérêts différents et, éventuellement, indiquer la voie à suivre et les instruments à mettre en oeuvre.

La plupart des participants ont tenté de définir la cohérence en constatant que les contradictions font partie de la vie, qu'elles en sont l'essence même. La vie sociale et politique est contradictoire et incohérente. La vie elle-même est pleine de contradictions. Est-il dès lors raisonnable de vouloir supprimer 
le maximum de contradictions? Faut-il vraiment rechercher la cohérence? En rendant transparentes les contradictions avec lesquelles nous vivons, nous parviendrons peut-être à les réduire par un processus dynamique et interactif.

Hésitations, malentendus mais aussi stéréotypes, sont les mots-clés qui ont surgi au cours du débat visant à mieux saisir l'essence de la cohérence. Dans le cas de l'Indonésie, pays en plein essor, une question se pose précisément de manière très concrète: comment harmoniser les instruments de l'aide au développement et de la promotion économique? Pour améliorer la cohérence, nous devons trouver des réponses qui dépassent les schémas traditionnels. La cohérence dans les relations extérieures implique que toutes les relations poursuivies avec un pays sont subordonnées à un objectif commun. Les actions individuelles ne doivent pas aller à l'encontre de cet objectif commun, même si les interventions des divers acteurs ne sont pas coordonnées entre elles). "La cohérence signifie, premièrement, que la main gauche sait ce que fait la main droite - ce qui correspond à la transparence - et, deuxièmement, qu'une action ne détruit pas le résultat des autres" (Violette Ruppanner). La recherche de la cohérence est donc un processus d'optimisation.

L'étude des conflits régionaux dans l'hémisphère Sud montre que les ruptures sont de plus en plus nombreuses et marquées. II convient dès lors d'évaluer avec soin les effets qu'une politique - à notre avis cohérente produit chez son destinataire. Dans le domaine socio-culturel, parvenir à surmonter les scissions et les conflits constitue un pas essentiel vers la démocratie, la prospérité économique, le respect des droits de l'homme et la protection de l'environnement. "Une politique qui aggraverait encore les conflits par exemple en favorisant un secteur donné ou certains acteurs, tout en oubliant les autres - serait incohérente" (Günther Bächler).

D'autres consultations devront déterminer qui définit l'objectif à atteindre les partenaires en Indonésie, les acteurs en Suisse, séparément ou en commun - et quel pourrait être cet objectif dans le cas de l'Indonésie. Deux points de vue s'opposent ici: “Le but qu'il faut viser consiste à élaborer une politique de développement globale et cohérente et, plus particulièrement, de mieux orienter les instruments économiques et de la politique commerciale sur les objectifs de la politique de développement" (Richard Gerster).Ce point de vue est toutefois en contradiction avec le principe libéral du "laisser-faire" préconisé par l'économie privée.

\section{Quel processus, quelles structures choisir?}

Dans un premier temps, on constate en Suisse un grand besoin d'agir, le besoin aussi de mettre en place un mécanisme d'information et de consultation systématiques. Un tel mécanisme pourrait améliorer la transparence en ce qui concerne les conflits entre objectifs. En Suisse, nous avons tendance à occulter les tensions et à vouloir donner une image harmonieuse en toutes choses. De plus, la politique de développement sert souvent à faire de la politique intérieure. Ces habitudes nous empêchent d'analyser clairement les 
antagonismes qui marquent la politique de développement et la politique étrangère. "Dans ce sens, je considère la cohérence comme un moyen pour la politique étrangère de se libérer de la politique intérieure" (Matthias Meyer).

Disposons-nous des structures nécessaires pour mener une politique cohérente? La politique économique est entre les mains d'un département, tandis que la politique extérieure et l'aide au développement relèvent d'un autre. Vue de l'extérieur, la coordination entre ces deux départements ne semble pas toujours optimale. II reste dès lors à savoir comment modifier les structures pour parvenir à un maximum de cohérence. A cette question, d'aucuns répliquent que la Suisse - avec sa tradition du consensus - possède justement les structures idéales pour formuler des politiques cohérentes. "La Suisse est le seul pays d'Europe occidentale où le consensus est si fortement enraciné. Les procédures administratives internes et notre culture de consultation extrêmement différenciée devraient offrir un cadre idéal à une politique cohérente" (Peter Maurer). Pourquoi ne parvenons-nous pas à la mettre en place? La réponse est à chercher dans le domaine politique. En effet, il n'y a guère que la Suisse qui sépare, depuis toujours et aussi nettement, la politique de l'économie. Sur le plan économique et financier, la Suisse est une grande puissance; sur le plan politique, elle s'est isolée du débat mondial. C'est pour cette raison qu'elle ne parvient pas à prendre les décisions correspondant à la qualité de ses structures.

"La cohérence signifie que les acteurs agissent à peu près de concert, sans que leurs actions doivent nécessairement être bien coordonnées. L'essentiel est que les efforts aillent dans la direction souhaitée. En effet, si ces efforts permettent à un pays d'avancer sur la voie du développement, les organisations de développement et les oeuvres d'entraide seront satisfaites, puisque la situation de chacun dans le pays peut s'améliorer. Elle ne s'améliore hélas pas forcément, car il reste encore tout le problème de la répartition des ressources" (Max Schieler).

Le colloque sur les relations de la Suisse avec I'Indonésie a davantage porté sur le niveau d'action en Suisse que sur ce que nous attendons de nos partenaires en Indonésie. Deux conclusions ressortent toutefois clairement: le besoin d'accroître la transparence et de mettre en place des instruments pour exercer une influence positive sur les décisions des différents acteurs dans les efforts de cohérence.

\section{La mise en oeuvre de la cohérence: quels mécanismes?}

L'objectif d'une plus grande cohérence des politiques dans la coopération au développement requiert des mécanismes efficaces de mise en oeuvre. Dans le cadre du colloque, cette question fut abordée sous plusieurs angles:

a) quel est le rôle de ces mécanismes (information, consultation, co-décision)?

b) quels sont les acteurs concernés par ces mécanismes?

c) les mécanismes existants suffisent-ils ou en faut-il de nouveaux? 


\section{a) Le rôle des mécanismes}

Un premier constat a fait rapidement l'unanimité: une bonne circulation de l'information est une condition nécessaire à la recherche d'une plus grande cohérence des politiques dans la coopération au développement. Dans le cas de la politique suisse de coopération au développement avec l'Indonésie, on a regretté que la décision de retrait de la DDA et du passage du témoin à I'OFAEE n'ait pas été discutée en temps opportun avec les milieux intéressés (ONG, économie privée).

L'accès des acteurs concernés à l'information sur les plans des autres acteurs permet de mieux identifier les contradictions possibles entre les politiques et les conflits d'intérêts des différents acteurs. La consultation permet donc de prendre des décisions en meilleure connaissance de cause tout en préservant l'autonomie des décisions de chaque acteur.

Faut-il aller au-delà de l'échange d'information et de la consultation jusqu'à un examen de la cohérence conduit conjointement par tous les acteurs (administration, ONG, économie privée)? C'est la thèse de Günther Bächler qui considère que la transparence n'est qu'une condition préalable pour la mise en place de mécanismes d'examen de la cohérence sous l'angle des différents objectifs de la politique suisse énoncés dans les lignes directrices. Cet examen de la cohérence ne concerne pas selon lui que l'Administration fédérale, mais aussi tous les autres acteurs: "Les différents acteurs devraient en principe effectués dans leur domaine spécifique cet éventail de contrôles de la compatibilité et parvenir à optimiser ces diverses stratégies ou projets en vue d'obtenir la cohérence."

D'autres participants estiment qu'il ne faut pas aller au-delà de la consultation: Selon Matthias Meyer, "c'est une chose que les milieux intéressés s'informent mutuellement de leurs plans et que cet échange facilite des prises de décisions. C'en est une autre que de vouloir exercer une influence sur les instances compétentes par le biais d'examens de la cohérence. Cet examen de la cohérence doit pour l'essentiel se faire au sein de l'Administration fédérale. Ce qu'il faut, c'est améliorer le processus de consultation des milieux privés.". Il ne faut pas non plus que la consultation dilue la responsabilité des instances appelées à prendre des décisions.

\section{b) Les acteurs}

Dans leurs contributions écrites au colloque, les participants ont identifié ou proposé des mécanismes différents selon les acteurs concernés. On y trouve d'une part les mécanismes internes à l'Administration fédérale et d'autre part les mécanismes impliquant d'autres acteurs, que ce soit en Suisse ou en Indonésie.

En ce qui concerne l'Administration fédérale, plusieurs participants extérieurs à cette administration ont exprimé le désir que la coordination entre les deux principaux offices fédéraux concernés, la DDA et l'OFAEE, soit renforcée, un intervenant allant jusqu'à suggérer une fusion du Service du développement 
de l'OFAEE et de la DDA. II est en tout cas jugé souhaitable et possible d'intégrer plus fortement les activités des deux offices fédéraux au sein des bureaux de coordination de la coopération suisse dans les pays de concentration de la coopération suisse au développement. Dans l'Administration fédérale, la coordination de la politique extérieure et de la politique économique extérieure devrait pouvoir se réaliser sans grande difficulté puisque, dans la plupart des pays en développement, la politique extérieure de la Suisse se confond pratiquement avec sa politique de coopération au développement.

La participation de l'économie privée aux mécanismes de concertation pose une question de principe. Pour Andreas Zürcher, "l'économie privée joue aujourd'hui un rôle important, mais son rôle dans la politique suisse de développement n'est pas défini". Tant qu'il n'est pas défini, les entreprises ne voient pas le sens de faire circuler de l'information. Comme elles jouent un rôle important dans les pays en développement, il s'agit de les impliquer dans le processus de recherche de cohérence. Pour ce faire, il est nécessaire que les autres acteurs reconnaissent le rôle que l'économie privée peut jouer dans le développement et que l'on laisse de côté les anciens différends et récriminations. Le dialogue sera difficile si le rôle de l'économie privée est constamment remis en cause et critiqué. II faut aussi se rendre compte que la réflexion au sein des entreprises sur le rôle qu'elles peuvent jouer dans la politique de développement n'est pas encore très avancée. Cependant, si ces conditions sont remplies et s'il existe un climat de confiance mutuelle, un véritable dialogue pourra avoir lieu car chaque acteur a de bonnes raisons de rechercher une plus grande cohérence.

Pour Philippe Régnier, il paraît particulièrement important de faire participer les petites et moyennes entreprises (PME) suisses à la coopération au développement avec l'Indonésie. Les PME suisses ne sont pas très actives sur les marchés extra-européens et, selon l'OCDE, la Suisse est en retard par rapport aux autres pays industriels pour ce qui est de leur promotion. Les efforts entrepris par des ONG telles que SWISSCONTACT ou FUNDES pour favoriser la promotion des PME dans les pays en développement n'ont pas rencontré un écho suffisant. Les instruments officiels de promotion devraient être évalués. Du côté de l'OFAEE, des projets sont à l'étude pour favoriser la formation, des "joint-ventures" et le transfert de technologies liées à la protection de l'environnement. En outre, une étude est en cours pour mettre sur pied une société suisse d'investissement pour le développement qui encouragerait des "joint-ventures" par des prêts, des participations au capital, ou l'octroi de garanties. Les milieux intéressés seront consultés.

La recherche de la cohérence doit aussi être poursuivie en intensifiant les contacts et le dialogue avec les partenaires en Indonésie. Avec les partenaires traditionnels de la Suisse (institutions publiques et ONG), un effort particulier devrait être entrepris pour mieux connaître ce qu'ils attendent des divers acteurs suisses. II convient aussi d'accorder une priorité à la recherche de nouveaux partenaires, en particulier, pour Robert Jenny, ceux qui émergent du processus de décentralisation qui est en cours en Indonésie 


\section{c) Quels mécanismes?}

Les mécanismes existants sont-ils suffisants ou faut-il en prévoir de nouveaux? Matthias Meyer est d'avis qu'il vaut mieux exploiter ce qui existe plutôt que de multiplier les lieux de concertation.

Le policy dialogue avec les partenaires indonésiens est un mécanisme dont l'utilité n'est pas contestée. Chaque rencontre peut offrir des occasions de dialoguer sur les thèmes qui paraissent importants aux divers acteurs des relations bilatérales. Les ONG sont déjà associées à la réflexion de l'Administration fédérale sur la politique suivie au sein des institutions de Bretton Woods. Une plus large inclusion des ONG dans le policy dialogue est en principe possible, mais du point de vue de l'Administration fédérale, il n'est pas toujours aisé de choisir les ONG avec lesquelles dialoguer; elles sont nombreuses et ont souvent des points de vue divergents.

Les chambres de commerce sont un instrument très utile de concertation car il est difficile de dialoguer dans un cadre ad hoc avec des entreprises individuelles. La Chambre de commerce pour l'Asie du Sud-Est pourra prendre en compte, de par son organisation, les questions propres à chacun des pays membres: mais les relations économiques ne sont pas suffisamment importantes pour permettre la survie d'une chambre de commerce purement bilatérale. La participation à cette Chambre de commerce de personnalités issues du monde universitaire et de l'Administration fédérale permettra à cette institution de servir de forum pour un dialogue sur la cohérence.

Les visites de conseillers fédéraux dans un pays de concentration de la coopération suisse ne devraient-elles pas représenter des "moments forts" de concertation avec l'ensemble des acteurs suisses? A propos de la visite du conseiller fédéral Delamuraz en Indonésie en octobre 1994, l'Union syndicale suisse aurait volontiers, selon Margrit Meier, communiqué quelques renseignements sur la situation des syndicats et le respect des droits de l'homme dans ce pays.

Parmi les propositions d'innovation présentées par certains des participants, deux ont été particulièrement discutées.

Pour Richard Gerster, il faudrait envisager l'introduction de "contrats de coopération symétriques, à long terme et cohérents qui définissent un cadre général pour toutes les dimensions des relations bilatérales. Actuellement, la Suisse conclut avec des pays en développement des accords portant sur les questions les plus diverses (double imposition, protection des investissement, trafic aérien, coopération au développement); la négociation d'un accord-cadre de coopération à long terme serait l'occasion de discuter avec les partenaires du pays de concentration non seulement tous les aspects de la politique de coopération, mais l'ensemble des questions bilatérales, en incluant par exemple le cas échéant les problèmes de migration." Cette proposition suscite des remarques critiques sur la difficulté de mettre en place un tel mécanisme ainsi que sur sa lourdeur possible. Quelques participants doutent que, dans le cas de l'Indonésie, la Suisse ait un poids suffisant pour pouvoir 
exercer une influence quelconque sur le pays partenaire. La masse critique est loin d'être atteinte. L'idée d'avoir avec un pays partenaire une négociation du type de celle que I'Union européenne a avec les pays partenaires des conventions de Lomé paraît difficilement réalisable de la part de la Suisse, sauf s'il s'agit un petit pays partenaire avec lequel les relations bilatérales sont particulièrement importantes. II a été observé que le cadre multilatéral permet aussi à la Suisse de se faire entendre des pays partenaires. Cependant, la Suisse ne peut exercer une influence sur la politique indonésienne que par le biais d'une conditionnalité bilatérale.

La proposition de créer une commission nationale tripartite (Administration fédérale, économie privée et universités) fonctionnant comme mécanisme de coordination et de collaboration fut accueillie avec intérêt; une telle commission pourrait aussi inclure les ONG. Mais quelques objections de principes ont été formulées. II faudrait disposer de commissions semblables pour tous les pays importants; cette prolifération de commissions pourrait-elle être gérée par les institutions concernées? Hans-Peter Maag s'est demandé si de telles commissions ne rendraient pas impossible la réduction ou la suppression de la coopération avec le pays concerné, alors que l'on demande par ailleurs à la DDA de concentrer davantage sa coopération.

\section{Conclusions}

Ce débat sur la cohérence fondé sur l'exemple de la coopération suisse avec un important pays est à considérer comme une première approche d'une problématique complexe. II est certain que la réflexion doit encore être approfondie tant sur le plan conceptuel que sur celui des mécanismes de mise en oeuvre. Il apparaît cependant aux responsables de ce dossier que, sur certains points importants, des conclusions peuvent être tirées qui indiquent le chemin à suivre pour progresser dans la mise en pratique de l'approche préconisée par le rapport du Conseil fédéral.

- La circulation de l'information, dans un forum ad hoc, sur les analyses et les projets des acteurs concernés par le développement économique et social d'un pays partenaire de la Suisse est nécessaire aussi bien pour la recherche de plus de cohérence dans les politiques que pour l'amélioration de la qualité des décisions des divers acteurs. Si les avis divergent quant à la possibilité ou l'opportunité d'aller plus loin dans l'examen de la cohérence des actions des différents acteurs, il est reconnu que de tels échanges sont nécessaires et tous les participants ont manifesté leur disponibilité pour y participer.

- Une condition nécessaire pour qu'un dialogue fructueux ait lieu est que le rôle de chacun dans la coopération au développement soit clairement défini. Ce n'est pas encore le cas aujourd'hui, en particulier pour ce qui est du rôle de l'économie privée II est important et urgent de combler cette lacune pour que progresse le dialogue sur la cohérence. 
- Une autre condition au renforcement de la réflexion sur la cohérence est le développement de la recherche et de la formation en Suisse sur l'Asie. Les futurs acteurs pourraient ainsi dès le début de leur carrière être informés des problèmes de développement de la région et être attentifs à la problématique de la cohérence. En tenant compte des ressources humaines et financières limitées, les milieux universitaires pourraient, d'entente avec les autres acteurs, établir un "cadre d'observation" qui recueillerait et rendrait pertinents pour les acteurs suisses les données et les résultats de recherches produits par les institutions spécialisées à travers le monde.

La conclusion la plus importante du colloque est sans doute que tous les acteurs suisses des relations avec l'Indonésie ont de bonnes raisons pour que la cohérence des politiques progresse. II leur reste maintenant à trouver le forum pour poursuivre la réflexion et à y associer de la façon la plus large possible les partenaires indonésiens.

L'Annuaire Suisse-Tiers Monde quant à lui sera toujours ouvert à des contributions sur ce thème. Ce premier examen des problèmes concrets que peut poser la mise en oeuvre de politiques plus cohérentes a en effet démontré que les acteurs que nous avions réunis reconnaissaient l'utilité de la démarche et y participaient dans un esprit d'ouverture, en laissant de côté les clichés et les idées reçues. 